\title{
INFERÊNCIA FUZZY PARA O PROBLEMA DE CORTE DE ESTOQUE COM SOBRAS APROVEITÁVEIS DE MATERIAL
}

\author{
Adriana Cristina Cherri ${ }^{1 *}$, Douglas José Alem Junior ${ }^{2}$ \\ e Ivan Nunes da Silva ${ }^{3}$
}

Recebido em dezembro 2008 / Aceito em julho 2010

\begin{abstract}
In this paper, we study the cutting stock problem with usable leftovers (CSPUL), that consists in determining a policy of usable leftovers from cut objects. These leftovers, if large enough, can returned to the stock as retails in order to fulfill future demands and, therefore, they are not computed as waste. In order to solve the CSPUL, several heuristic procedures from the literature can be used. However, it is hard to point out which solution method performs better, since each one presents different solutions regarding some conflicting characteristics, such as quantity of new retails generated, wastes, and distribution of the wastes and retails in the cut objects. To overcome this difficulty, we propose some fuzzy techniques to consider the contribution of each characteristic in the solution, classify it according some criteria beforehand considered and, finally, identify the best solution method based on the respective solution. With the proposed fuzzy methodology, the solution methods analysis was simplified.
\end{abstract}

Keywords: cutting stock problems, usable leftovers, fuzzy inference.

RESUMO. Nesse trabalho, é estudado o problema de corte de estoque com sobras aproveitáveis (PCESA), que consiste em determinar uma política de aproveitamento das sobras de objetos cortados. Estas sobras, desde que não sejam demasiadamente pequenas, podem retornar ao estoque como retalhos para atender demandas futuras, não sendo, portanto, consideradas perdas. Para resolver o PCESA, podem ser utilizados vários procedimentos heurísticos propostos na literatura. Entretanto, é difícil identificar o melhor método de solução, pois cada um deles apresenta uma solução diferente em relação às características de interesse do problema, como quantidade de retalhos produzidos, perdas e distribuição de perdas e retalhos nos objetos. Para superar essa dificuldade, propõe-se utilizar técnicas da lógica fuzzy para considerar a contribuição de cada característica de interesse na solução, classificá-la de acordo com critérios pré-estabelecidos e, finalmente, identificar o melhor método com base na respectiva solução. Com a metodologia fuzzy desenvolvida, facilitou-se a análise dos métodos de solução.

Palavras-chave: problemas de corte de estoque, sobras aproveitáveis, inferência fuzzy.

\footnotetext{
*Autor correspondente

${ }^{1}$ Departamento de Matemática, Universidade Estadual Paulista Júlio de Mesquita Filho, Av. Eng. Luiz Edmundo Carrijo Coube, 14-01, Vargem Limpa, 17033-360 Bauru, SP, Brasil. E-mail: adriana@fc.unesp.br

2 Engenharia de Produção, Universidade Federal de São Carlos, campus de Sorocaba, Rodovia João Leme dos Santos, Km 110, SP-264, Bairro do Itinga 18052-780 Sorocaba, SP, Brasil. E-mail: douglas@ufscar.br

${ }^{3}$ Departamento de Engenharia Elétrica, EESC, Universidade de São Paulo, Av. Trabalhador São-carlense, 400, Caixa Postal 473, 13560-970 São Carlos, SP, Brasil. E-mail: in silva@sel.eesc.usp.br
} 


\section{INTRODUÇÃO}

Os problemas de corte de estoque (PCE) aparecem em diversos processos industriais, em que os objetos a serem cortados correspondem a barras de aço, bobinas de papel e alumínio, placas metálicas e de madeira, placas de circuito impresso, chapas de vidro, peças de couro, entre outros. Nesses contextos, a redução dos custos de produção e a melhoria da eficiência do processo produtivo estão frequentemente associadas à utilização de estratégias adequadas dos processos de corte. O leitor interessado em problemas de corte de estoque pode consultar os seguintes trabalhos: Hinxman (1980), Dyckhoff et al. (1985), Dyckhoff \& Wäscher (1990), Dowsland \& Dowsland (1992), Sweeney \& Paternoster (1992), Bischoff \& Wäscher (1995), Dyckhoff et al. (1997), Arenales et al. (1999), Wang \& Wäscher (2002), Hifi (2002), Oliveira \& Wäscher (2007) e Wäscher et al. (2007). Referências adicionais podem ser encontradas em ESICUP - European Interested group in CUtting and Packing Problems (2009).

Um problema pouco estudado e comumente encontrado na prática consiste em determinar uma política de aproveitamento das sobras de objetos cortados. Estas sobras, desde que não sejam demasiadamente pequenas, podem retornar ao estoque como retalhos para atender demandas futuras e, portanto, não são consideradas perdas. Esse problema é frequentemente denominado de problema de corte de estoque com sobras aproveitáveis (PCESA).

O PCESA foi identificado no trabalho de Brown (1971), entretanto, os primeiros trabalhos envolvendo o PCESA surgiram com o trabalho de Dyckhoff (1981), no qual o autor desenvolveu um modelo para o PCE caracterizado pelo uso dinâmico de padrões de corte estruturados. O modelo apresenta vantagens de uso em situações práticas em que os objetos em estoque possuem comprimentos diferentes e existe um número relativamente grande de itens demandados. As sobras geradas pelo processo de corte têm um valor associado, pois podem ser utilizadas para atender novas demandas que surgem ao longo do horizonte de planejamento considerado. Outras estratégias para lidar com o PCESA podem ser encontradas em Roodman (1986), Scheithauer (1991), Sinuany-Stern \& Weiner (1994), Gradisar et al. (1997), Gradisar et al. (1999) e Abuabara \& Morabito (2009).

Recentemente, Cherri et al. (2009) desenvolveram um estudo sobre o aproveitamento de sobras durante o processo de corte e propuseram modificações em métodos heurísticos tradicionais para lidar com o problema de sobras aproveitáveis. Os autores mostraram a dificuldade em identificar o melhor método para o PCESA, pois cada um deles apresentou uma solução diferente em relação às características de interesse do problema, como quantidade de retalhos produzidos, perdas e distribuição de perdas e retalhos nos objetos.

Nesse contexto, o presente trabalho propõe a utilização de algumas técnicas da lógica fuzzy para auxiliar na escolha do melhor método para resolver o PCESA proposto em Cherri et al. (2009). Em linhas gerais, a abordagem proposta leva em consideração a contribuição de cada característica de interesse na solução, classifica as soluções de acordo com critérios pré-estabelecidos e, finalmente, identifica o melhor método com base na respectiva solução. Convém salientar que até a finalização desse trabalho, não foi encontrada nenhuma técnica geral capaz de comparar 
métodos de solução quando vários fatores conflitantes concorrem na determinação do melhor método. O conhecido trabalho de Dolan e Moré (2002), por exemplo, propõe a utilização de perfis de desempenho como uma poderosa técnica na comparação de softwares científicos (ou métodos de solução). Porém, a comparação é realizada com base em apenas uma métrica (ou característica de interesse), como tempo de resolução necessário para obter uma solução com determinada acurácia, por exemplo. Assim, esta metodologia não pode ser aplicada diretamente no caso proposto por esse artigo.

A teoria dos conjuntos fuzzy, introduzida por Zadeh (1965), surgiu como um meio de representação e manipulação de dados imprecisos, e pelo fato de os conjuntos clássicos apresentarem limitações para lidar com problemas em que transições de uma classe para outra acontecem de forma lenta e gradual. Nos últimos anos, a lógica fuzzy tem sido empregada em diversos problemas gerais de pesquisa operacional, como localização de facilidades, logística e transporte, designação, tomada de decisão em ambientes incertos, sequenciamento, entre outros (Guiffrida $\&$ Nagi, 1998). Particularmente para os problemas de corte de estoque, a lógica fuzzy foi empregada no trabalho de Vasko et al. (1989), que apresentaram uma formulação matemática para o PCE em um ambiente fuzzy. O problema de corte de estoque fuzzy definido pelos autores tem o objetivo de determinar padrões de corte sujeito a restrições sobre a produtividade do processo de corte, serviços de clientes e logística. Ghodsi \& Sassani (2002) desenvolveram um algoritmo fuzzy para minimizar a perda no corte de placas de madeira para a produção de peças menores. Ghodsi \& Sassani (2005), apresentaram um método de classificação fuzzy para a otimização do corte de estoque em tempo real com ordem de prioridades. Mais recentemente, Saad et al. (2007) propuseram a utilização da lógica fuzzy para modelar um PCE com múltiplos objetivos em uma indústria de alumínio.

O restante desse artigo está organizado da seguinte maneira: na Seção 2, é definido o problema de corte de estoque com sobras de material aproveitáveis. Na Seção 3, são revisados alguns métodos heurísticos propostos por Cherri et al. (2009) para obter a solução do PCESA. Na Seção 4, é realizada uma revisão sobre estimadores e controladores fuzzy. Resultados computacionais são apresentados na Seção 5. Na Seção 6, foi realizada a análise das soluções a partir da técnica de inferência fuzzy. Finalmente, na Seção 7, são apresentadas as conclusões e sugestões de trabalhos futuros.

\section{O PROBLEMA DE CORTE DE ESTOQUE UNIDIMENSIONAL COM SOBRAS DE MATERIAL APROVEITÁVEIS}

Os métodos existentes na literatura para resolver problemas de corte de estoque unidimensionais têm como principal objetivo minimizar a sobra resultante nos objetos cortados. Para os problemas de corte, as modelagens matemáticas e os métodos de resolução de maior impacto na literatura foram publicados por Gilmore \& Gomory $(1961,1963)$.

- Definir todos os possíveis padrões de corte (maneira como um objeto em estoque é cortado para a produção de itens demandados).

- Definir o número de vezes que cada padrão de corte é utilizado para atender a demanda. 
Os problemas de corte de estoque aparecem em diversas situações e em cada uma delas, há especificidades, restrições e objetivos impostos pelas práticas industriais que devem ser respeitados. Um problema que tem recebido atenção especial nos últimos anos consiste em aproveitar sobras de padrões de corte desde que estas não sejam pequenas. A idéia de aproveitar sobras de padrões de corte é uma realidade em muitas empresas, principalmente quando o custo da matéria-prima (barras ou placas de diferentes materiais) representa uma parte significativa do custo do produto final. Além do apelo econômico evidente do processo de aproveitamento das sobras de material, há ainda a motivação da sustentabilidade em seus diversos âmbitos (social, econômico e ambiental) que tornam esse problema importante e desafiador.

Nesse trabalho, não é apresentada uma formulação matemática para o problema de corte de estoque com sobras aproveitáveis. Porém, como a qualidade dos padrões de corte depende diretamente dos comprimentos e quantidades de itens a serem produzidos, os padrões de corte podem ser planejados de modo a admitir sobras grandes (retalhos) que possam ser utilizadas no futuro. Assim, é possível desenvolver métodos heurísticos que, a partir de um conjunto de dados de entrada e objetivos definidos, obtenha tais conjuntos de padrões de corte, tendo em vista o aproveitamento das sobras do processo de corte de estoque. De acordo com Cherri et al. (2009), o problema de corte de estoque com sobras aproveitáveis é definido da seguinte maneira:

"Um conjunto de itens deve ser produzido a partir do corte de objetos, os quais podem ser de tamanhos padronizados (objetos que são comprados de fornecedores) ou não padronizados (objetos que são retalhos de cortes anteriores). São conhecidos o comprimentos e as quantidades dos itens demandados, assim como as características dos objetos disponiveis no estoque. As demandas devem ser atendidas cortando-se os objetos disponíveis, de modo que as sobras geradas sejam 'pequenas' (chamadas de perda) ou 'suficientemente grandes' (chamadas de retalhos) para retornarem ao estoque, porém em número reduzido".

Existem várias maneiras de se definir um comprimento 'suficientemente grande' ou mínimo aceitável para um retalho (Abuabara \& Morabito, 2009; Gradisar et al., 1997; Cherri et al., 2009). Neste artigo, o mínimo aceitável para um retalho $(\delta)$ é definido como a média dos comprimentos dos itens demandados. Os parâmetros $\theta$ e $\beta$ são utilizados pelo usuário para definir o tamanho máximo para que uma sobra possa ser considerada uma perda pequena. Desta forma, tem-se:

- $\theta$ : fração que define uma perda pequena para os objetos padronizados e $\theta L_{k}$ é o comprimento máximo para uma sobra ser considerada perda pequena $(k=1, \ldots, \bar{k}$, em que $\bar{k}$ é a quantidade de tipos de objetos padronizados em estoque).

- $\beta$ : fração que define uma perda pequena para os objetos não padronizados e $\beta L_{k}$ é o comprimentos máximo para que uma sobra seja considerada perda pequena $(k=\bar{k}+$ $1, \ldots, K$, em que os objetos do tipo $\bar{k}+1, \ldots, K$ são retalhos que retornaram ao estoque).

As sobras dos padrões de corte que forem maiores que $\theta L_{k}$ e $\beta L_{k}$ e menores que $\delta$ são consideradas como perda de tamanho intermediário. A Figura 1 ilustra a classificação das sobras geradas em um objeto. 


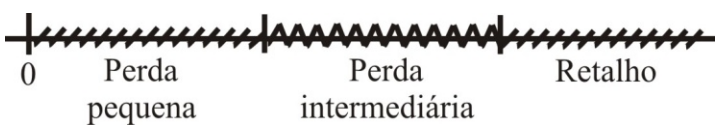

Figura 1 - Classificação das sobras.

Pela definição do PCESA, observa-se que alguns objetivos nem sempre são satisfeitos simultaneamente, pois é possível obter soluções com sobras de tamanho intermediário e retalhos distribuídos em muitos objetos cortados, ou soluções com sobras de tamanho intermediário e retalhos concentrados em poucos objetos cortados.

Em Cherri et al. (2009), as soluções para o PCESA foram classificadas como ideal, aceitável e indesejável, sendo que o número de objetos cortados com perda pequena, perda de tamanho intermediário e retalhos influenciam diretamente nessa classificação. Nesse trabalho, as soluções são definidas da seguinte maneira:

Definição 1: Para o PCESA, as soluções são definidas como:

- Solução ideal: se a redução de retalhos (objetos não padronizados) for bem significativa e a perda relativa nos objetos padronizados e não padronizados for muito pequena.

- Solução desejável: se a redução de retalhos for bem significativa, a perda relativa nos objetos padronizados for muito pequena e a perda relativa nos objetos não padronizados for pequena.

- Solução aceitável: se a redução de retalhos for pouco significativa e a perda nos objetos padronizados for pequena.

- Solução indesejável: se o estoque de retalhos manter-se estável e a perda relativa nos objetos padronizados for pequena.

- Solução inaceitável: se o estoque de retalhos ampliar-se.

Na Definição 1, as soluções são classificadas considerando cinco categorias. Entretanto, outras categorias poderiam ser definidas. Esta nova classificação requer a quantificação dos termos muito pequena e pequena, redução bem significativa, pouco significativa, estável ou ampliada. Para isso, definiu-se:

- muito pequena: se perda relativa $<\xi_{1}^{p}\left(\xi_{1}^{n p}\right)$.

- pequena: se perda relativa $<\xi_{2}^{p}\left(\xi_{2}^{n p}\right)$.

sendo que $0<\xi_{1}^{p}<\xi_{2}^{p}<1$ é o limite da perda relativa nos objetos padronizados e $0<\xi_{1}^{n p}<$ $\xi_{2}^{n p}<1$ é o limite da perda relativa nos objetos não padronizados.

A perda relativa é calculada pela razão entre a perda total e o comprimento dos objetos cortados:

$$
\text { Perda relativa }=\frac{\text { comprimento total perdido }}{\text { comprimento total de objetos cortados }},
$$


em que o comprimento perdido e o comprimento total dos objetos cortados devem ser referentes ao mesmo tipo de objeto (padronizado ou não padronizado).

Para obter as variações do estoque de objetos não padronizados (retalhos), calcula-se o retalho líquido relativo:

Retalho líquido relativo $=\frac{\text { número de objetos retalho em estoque }- \text { retalhos utilizados }+ \text { novos retalhos }}{\text { número de objetos retalho em estoque }}$

no qual o número de objetos retalho em estoque é a quantidade de objetos não padronizados em estoque antes de iniciar o processo de corte. Note que o retalho líquido mostra o balanço do estoque de retalhos. Sendo assim, é natural que a sua redução seja uma medida de interesse para o PCESA.

Para a quantificação dos termos referentes ao retalho líquido relativo, define-se a redução do estoque da seguinte maneira:

- bem significativa: se retalho líquido relativo $\leq \omega_{1}$;

- pouco significativa: se $\omega_{1}<$ retalho líquido relativo $\leq \omega_{2}$;

- estável: se $\omega_{2}<$ retalho líquido relativo $\leq \omega_{3}$;

- ampliado: se retalho líquido relativo $>\omega_{3}$,

em que os parâmetros $\omega_{1}, \omega_{2}$ e $\omega_{3}$ também são definidos pelo usuário, de forma que $0<\omega_{1}<$ $\omega_{2}<\omega_{3} \leq 1$.

Convém ressaltar que o uso do retalho líquido na análise das soluções só deve ser utilizado quando existir uma determinada quantidade de objetos em estoque (pelo menos maior do que 1). Este critério não deve ser utilizado quando, por exemplo, o estoque de retalhos é nulo, pois se apenas um retalho for gerado durante o processo de corte, o retalho líquido relativo será infinito.

Para compreender a dificuldade em analisar as soluções do PCESA, considere o exemplo a seguir em que $d_{i}, i=1, \ldots, m$ é a demanda dos itens e $e_{k}, k=1, \ldots, K$, é a disponibilidade dos objetos em estoque. Neste exemplo ilustrativo, assume-se que toda sobra superior a $4 \mathrm{~m}$ (média do comprimento dos itens demandados) é considerada retalho.
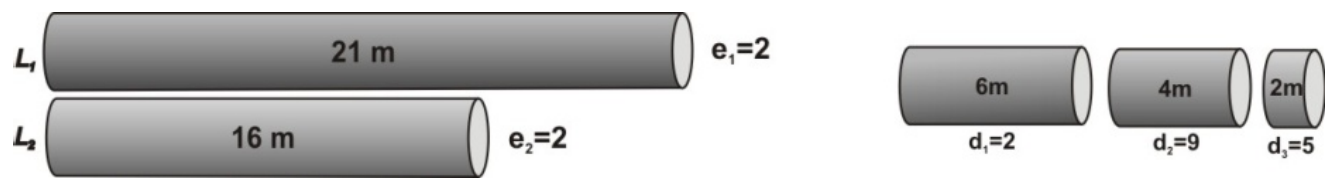

Figura 2 - Dados de um problema de corte.

Nas três possíveis soluções apresentadas tem-se a mesma sobra total, entretanto, a maneira como estas sobras estão distribuídas nos padrões de corte nos leva à dúvida da melhor solução, pois cada uma delas apresenta uma característica diferente em termos de comprimento e quantidade perdida e quantidade de novos retalhos que podem retornar ao estoque. Na solução (a) (Fig. 3), 


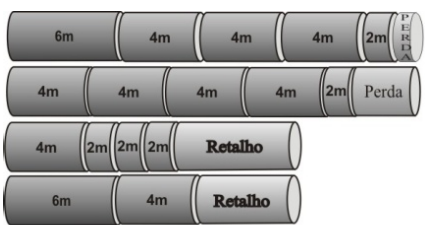

(a)

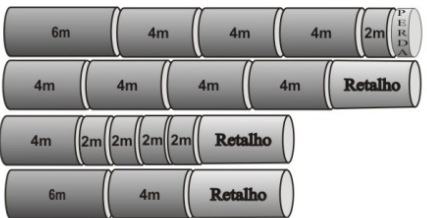

(b)

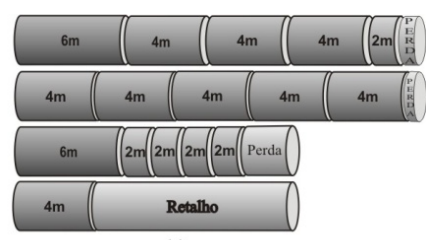

(c)

Figura 3 - Soluções alternativas para o PCESA.

por exemplo, dois retalhos foram gerados, porém, a perda total desta solução é maior do que a perda total da solução (b), que gerou 3 novos retalhos. Embora a solução (c) apresente uma perda total maior do que as demais soluções, ela gera um único retalho para estoque. Como se pode observar, a escolha da melhor solução não é trivial, pois a relação entre perda, retalho, quantidade de padrões com perda ou retalho podem ser conflitantes, tornando a análise das soluções difícil.

Para contornar esta dificuldade, este trabalho propõe a utilização de uma abordagem fuzzy para analisar e qualificar as soluções do PCESA obtidas através dos procedimentos heurísticos desenvolvidos por Cherri et al. (2009). Embora vários procedimentos heurísticos tenham sido desenvolvidos para resolver o PCESA, comparou-se apenas as soluções das heurísticas FFD (First Fit Decreasing), $\mathrm{FFD}_{\mathrm{A}}$, RAG (Residual de Arredondamento Guloso), $\mathrm{RAG}_{\mathrm{A}}$ e o algoritmo COLA, desenvolvido por Gradisar et al. (1997).

\section{HEURÍSTICAS PARA O PCESA}

Os métodos propostos para resolver o PCESA consistem em modificações de procedimentos heurísticos clássicos da literatura. Nesta seção, são revisadas as alterações realizadas nas heurísticas clássicas FFD e RAG versão 2, que originaram as heurísticas de aproveitamento $\mathrm{FFD}_{\mathrm{A}}$ e $\mathrm{RAG}_{\mathrm{A}}$ versão 2. Os detalhes destas heurísticas, bem como seus respectivos algoritmos, encontram-se em Cherri et al. (2009). Por simplicidade de notação, as heurísticas RAG e RAG versão 2 são denominadas apenas RAG e RAG $_{\mathrm{A}}$.

\subsection{Heurística FFD $_{\mathrm{A}}$}

A heurística $\mathrm{FFD}_{\mathrm{A}}$ consiste em aplicar o procedimento FFD para obter um padrão de corte e analisar a sobra obtida. Se a sobra for aceitável (por simplicidade, utiliza-se o termo sobra aceitável quando esta for uma perda pequena ou um retalho), o padrão é aceito. Senão, o maior item do padrão é retirado. Assim, para o espaço gerado com a retirada do item é resolvido o problema da mochila (Cherri et al., 2009) cuja capacidade é a sobra no padrão adicionada ao tamanho do item retirado. Depois de resolvido o problema da mochila, a sobra gerada é analisada e se não for aceitável, o segundo maior item do padrão é retirado. Novamente, para o espaço gerado, é resolvido o problema da mochila. Caso se tenha retirado um item de cada comprimento dentre todos que compõem o padrão de corte, volta-se a retirar o primeiro maior item. Este procedimento é repetido até que a sobra seja aceitável ou o padrão inicial tenha sido anulado. Neste último caso, o padrão de corte é gerado pelo problema da mochila. 


\subsection{Heurística RAG $_{\mathrm{A}}$}

A Heurística $\mathrm{RAG}_{\mathrm{A}}$ consiste em encontrar uma solução inteira aproximada a partir da solução da relaxação linear do problema proposto por Gilmore \& Gomory (1963):

$$
\begin{array}{lll}
\text { Minimizar } & f(\mathbf{x})=\sum_{k=1}^{K} \sum_{j=1}^{N_{k}} c_{j k} x_{j k} & \\
\text { sujeito a : } & \sum_{k=1}^{K} \sum_{j=1}^{N_{k}} \alpha_{i j k} x_{j k}=d_{i}, \quad i=1, \ldots, m \\
& \sum_{j=1}^{N_{k}} x_{j k} \leq e_{k}, & k=1, \ldots, K \\
& x_{j k} \geq 0 \text { e inteiro } & j=1, \ldots, N_{k}, k=1, \ldots, K
\end{array}
$$

utilizando a heurística RAG (Poldi \& Arenales, 2009). Depois de gerados todos os padrões de corte com a heurística RAG, a sobra em cada padrão é analisada. Se a sobra estiver em limitantes aceitáveis (calculados previamente), o padrão de corte analisado é aceito e armazenado; caso contrário, é rejeitado e em seguida desfeito (demanda e estoque são atualizados). Depois de analisados todos os padrões de corte gerados, aplica-se a heurística $\mathrm{FFD}_{\mathrm{A}}$ na demanda residual formada pelos padrões de corte rejeitados.

\section{TÉCNICAS DE INFERÊNCIA FUZZY}

A lógica Fuzzy é baseada na teoria dos conjuntos fuzzy. Esta é uma generalização da teoria dos conjuntos tradicionais para resolver os paradoxos gerados a partir da classificação "verdadeiro ou falso" da lógica clássica. Tradicionalmente, uma proposição lógica tem dois extremos: ou "completamente verdadeira" ou "completamente falsa". Entretanto, na lógica fuzzy, uma premissa varia em grau de verdade de 0 a 1 , tornando-a parcialmente verdadeira ou parcialmente falsa.

Para Pedrycz \& Gomide (1998), o uso de conjuntos fuzzy produz uma base para um meio sistemático para a manipulação das concepções incertas e vagas. Em particular, pode-se empregar conjuntos fuzzy para representar as variáveis linguísticas. Uma variável linguística pode ser considerada como qualquer variável cujo valor é um número fuzzy (os números fuzzy são constituídos de conjuntos fuzzy, definidos em universos de discurso discretos ou contínuos) ou cujos valores são definidos em termos linguísticos. As principais operações entre variáveis linguísticas são realizadas através da utilização dos conectivos "e" (operador de intersecção $\tau$-norma), "ou" (operador de intersecção $S$-norma) e "não". Assim, dados dois termos $A$ e $B$ de uma determinada variável linguística, as operações compostas por " $A$ e $B$ " e " $A$ ou $B$ ", são definidas em termos dos seus graus de pertinência como:

$$
\mu_{A}(x) \text { e } \mu_{B}(x)=\mu_{A}(x) \tau \mu_{B}(x) \text { e } \mu_{A}(x) \text { ou } \mu_{B}(x)=\mu_{A}(x) S \mu_{B}(x),
$$

em que $x \in U$ (universo de discurso). 
Utilizando-se para o operador $\tau$-norma a função mínimo (min) e para o operador $S$-norma a função máximo (max), tem-se:

$$
\mu_{A}(x) \text { e } \mu_{B}(x)=\min \left(\mu_{A}(x), \mu_{B}(x)\right), \quad \mu_{A}(x) \text { ou } \mu_{B}(x)=\max \left(\mu_{A}(x), \mu_{B}(x)\right) .
$$

Para a operação de complemento "não", tem-se a seguinte expressão:

$$
\text { não }\left(\mu_{A}(x)\right)=1-\mu_{A}(x), \quad x \in U \text {. }
$$

Se $x$ e $y$ forem variáveis linguísticas compostas respectivamente por um conjunto de termos $\left\{A_{1}, A_{2}, \ldots, A_{n}\right\}$ e $\left\{B_{1}, B_{2}, \ldots, B_{m}\right\}$ o problema básico do processo de inferência é encontrar uma função de pertinência $B^{\prime}$ que representa a consequência da aplicação simultânea das regras da forma "se-então".

Normalmente, os processos de inferência fuzzy aplicados em determinadas regras são baseados na regra de Modus Ponens generalizada que é explicitada para um conjunto observável $A^{\prime}$ como:

- Fato: $x$ é $A^{\prime}$;

- Regra: se $x$ é $A$ então $y$ é $B$;

- Consequência: $y$ é $B^{\prime}$.

Logo, se o conjunto $A$ implica diretamente no conjunto $B(A \rightarrow B)$, então esta operação de implicação pode ser transformada em uma relação de implicação $R_{A \rightarrow B}(x, y)$. Desta forma, para obtermos o conjunto $B^{\prime}$, basta compor o conjunto que denota um fato observável $A^{\prime}$ com a relação de implicação $R_{A \rightarrow B}(x, y)$ utilizando a operação de composição max-min (Pedrycz \& Gomide, 1998), ou seja:

$$
B^{\prime}=A^{\prime}(x) \circ R_{A \rightarrow B}(x, y)
$$

\subsection{Relações de implicação}

A obtenção da função de pertinência relativa à relação de implicação $R_{A \rightarrow B}$ pode ser computada utilizando vários operadores de implicação (Pedrycz \& Gomide, 1998). Considerando as variáveis linguísticas $A$ e $B$, a função de pertinência $\mu_{R_{A \rightarrow B}}(x, y)$ pode ser obtida, por exemplo, através do operador de Mandami, cuja idéia é descrever determinados processos por meio de variáveis linguísticas e usar estas variáveis como entrada para regras de controle. Formalmente, tem-se:

$$
\mu_{R_{A \rightarrow B}}(x, y)=\min \left(\mu_{A}(x) ; \mu_{B}(y)\right) .
$$

Definição 2: Singleton é um caso particular de conjunto fuzzy normalizado, cujo suporte é um único ponto $x \in X \operatorname{com} \mu(x)=1$.

Os conjuntos singleton são especialmente utilizados para mapear as grandezas de entrada do sistema fuzzy que geralmente são representadas por valores pontuais. 


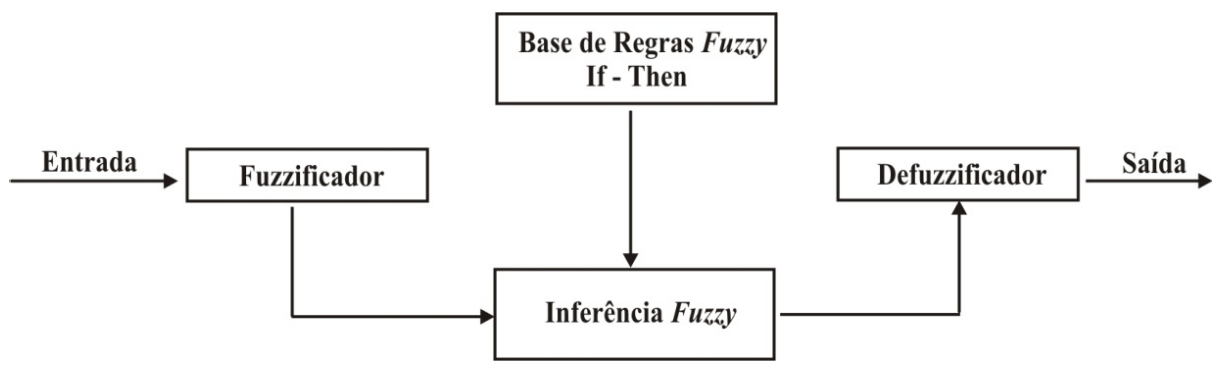

Figura 4 - Sistema fuzzy.

De acordo com Wang (1996), genericamente, um sistema fuzzy é composto de quatro componentes conectados:

Fuzzificadores - A principal função de um fuzzificador é converter os valores reais de entrada em um grau de pertinência a conjuntos fuzzy. Dentre todos os fuzzificadores existentes, um dos mais utilizados é o singleton.

Base de regras fuzzy - A base de regras fuzzy consiste de um conjunto de regras linguísticas "IF-THEN", sendo considerada a parte fundamental de um sistema fuzzy, uma vez que todos os outros componentes são utilizados para implementar as regras de modo eficiente e razoável.

Inferência $f u z z y$ - Nesta etapa, as proposições (regras) são definidas e depois são examinadas paralelamente. No processo de inferência fuzzy, os princípios da lógica fuzzy são usados para combinar as regras fuzzy "IF-THEN" (proposições condicionais) existentes na base de regras com os dados fuzzy de entrada. O resultado final desta combinação é uma região fuzzy de saída que está relacionada com a saída do processo. Os passos para o alcance dessa região fuzzy de saída são constituídas por:

- Encontrar todas as regras que estejam ativadas num determinado momento.

- Determinar a saída fuzzy de cada uma das regras ativadas.

- Combinar (agregar) todas as saídas fuzzy calculadas.

Assim, se for considerado um sistema composto por duas entradas e uma saída, com as variáveis linguísticas de entrada $x$ e $y$ compostas por um conjunto de termos fuzzy $\left\{A_{1}, A_{2}, \ldots, A_{n}\right\}$, $\left\{B_{1}, B_{2}, \ldots, B_{m}\right\}$ e uma variável de saída $z$ especificada no conjunto de termos $\left\{C_{1}, C_{2}, \ldots\right.$, $\left.C_{p}\right\}$, tem-se:

- Fato 1: $x$ é $A^{\prime}$;

- Fato 2: $y$ é $B^{\prime}$;

- Regra 1: se ( $x$ é $\left.A_{1}\right)$ e ( $y$ é $\left.B_{1}\right)$ então $z$ é $C_{1}$;

- Regra 2: se ( $x$ é $A_{2}$ ) e ( $y$ é $B_{2}$ ) então $z$ é $C_{2}$;

- Consequência: $z$ é $C^{\prime}$. 
Para se obter a relação de implicação $R_{A}$ e $B \rightarrow C$, basta aplicar o conectivo lógico "e" em todas as regras ativadas, levando-se em consideração somente a relevância em termos do grau de ativação e, em seguida, detectar todas as regras ativadas e suas respectivas regiões fuzzy de saída. Utilizando a operação de composição max-min, tem-se para a $k$-ésima regra ativada a relação:

$$
C_{k}^{\prime}(z)=A B^{\prime}(x, y) \circ R_{A B \rightarrow C}(x, y, z) .
$$

Combinando todas as regiões fuzzy de saída $C_{k}^{\prime}(z)$, obtém-se uma região fuzzy $C(z)$ que representa a agregação (denotada por $A g$ ) de todas as contribuições $C_{k}^{\prime}(z)$, ou seja, $C_{k}^{\prime}=$ $A g\left(C_{1}^{\prime}, C_{2}^{\prime}, \ldots, C_{k}^{\prime}\right)$, em que $k$ é o número de regras ativadas.

A agregação é a forma de combinar os consequentes (conjuntos fuzzy de saída) gerados a partir das regras ativadas. Normalmente, faz-se a agregação pelo máximo, ou seja, entre os diversos consequentes de um mesmo conjunto gerados na implicação, seleciona-se o de maior grau de pertinência.

Defuzzificadores - O defuzzificador especifica um ponto na saída que melhor representa o conjunto fuzzy. Na escolha de um defuzzificador, os critérios de plausibilidade, simplicidade computacional e de continuidade devem ser considerados. Segundo Wang (1996) os defuzzificadores mais utilizados são: centro de área, média dos máximos e primeiro máximo.

\subsection{Métodos de defuzzificação}

Os métodos de defuzzificação são frequentemente baseados em ideias heurísticas. Entretanto, esses métodos também podem ser caracterizados por suas propriedades (matemáticas) formais. A defuzzificação não é somente relevante para controles fuzzy, mas também para outros tipos de problemas.

Para determinar a região fuzzy gerada por todas as regras ativadas, deve-se aplicar um método de defuzzificação no conjunto $C^{\prime}(z)$ resultante da agregação de todos os conjuntos fuzzy de saída $C_{k}^{\prime}(z)$. Dentre os vários métodos definidos para o processo de defuzzificação, utiliza-se o centro de área (CDA), cuja ideia é agregar as informações sobre possíveis ações de controle que são representadas pela função de pertinência. A solução é um termo comum que combina a contribuição de cada regra ativada das consequências. Formalmente, tem-se:

$$
C D A=\frac{\sum_{k=1}^{n} \mu_{C^{\prime}}\left(V_{k}\right) V_{k}}{\sum_{k=1}^{n} \mu_{C^{\prime}}\left(V_{k}\right)},
$$

em que $n$ é o número de discretizações realizadas no universo de discurso de $C^{\prime}$ e $V_{k}$ são os valores do universo de discurso de $C^{\prime}$. 


\section{EXPERIMENTOS COMPUTACIONAIS}

\subsection{Classes de exemplos}

Para avaliar os procedimentos heurísticos descritos na Seção 3, 18 classes de exemplos com 50 exemplares cada foram gerados aleatoriamente com base no gerador apresentado em Cherri et al. (2009), que considera:

- Numero de tipos de objetos padronizados: $\bar{k}=2$.

- Número de tipos de objetos não padronizados: $k=3,5$ e 7 .

- Número total de objetos disponíveis em estoque: $K=5,7$ e 9 .

- Número de tipos de itens: $m=10,20$ e 40.

- Disponibilidades em estoque dos objetos padronizados: $e_{1}=e_{2}=100$ objetos.

- Disponibilidades em estoque dos objetos não padronizados: $e_{k}, k=3, \ldots, K$ são gerados aleatoriamente no intervalo $[1,10]$.

- Comprimentos dos objetos padronizados: $L_{1}=900$ e $L_{2}=1100$.

- Comprimentos dos itens: os itens de comprimento $\ell_{i}$ são gerados aleatoriamente no intervalo $\left[v_{1} L, v_{2} L\right.$ ], em que $L$ é o valor médio de $L_{k}, k=1,2, v_{1}=0,001, v_{2}=0,25$ (para itens de comprimento pequenos $(P))$ e 0,5 (para itens de comprimento variados $(V)$ ).

- Tamanho aceitável para o retalho: $\delta=\left\lfloor\frac{1}{m} \sum_{i=1}^{m} \ell_{i}\right\rfloor$.

- Comprimento dos objetos não padronizados: $L_{k}, k=3, \ldots, K$ são gerados aleatoriamente no intervalo de $\left[\delta, \frac{L_{1}}{2}\right]$.

- Demanda: $d_{i}$ é gerada aleatoriamente no intervalo $[1,5]$.

- Tamanho máximo de perda pequena para os objetos padronizados: $0,005 L_{k}, k=1,2$.

- Tamanho máximo de perda pequena para os objetos não padronizados: $0,05 L_{k}, k=$ $3, \ldots, K$.

Note que a demanda dos itens é baixa, o que reflete uma situação frequente em indústrias de pequeno porte (Abuabara \& Morabito, 2009). Nos exemplares gerados, definiu-se uma porcentagem de perda aceitável maior para os objetos não padronizados para que estes tenham maior chance de serem utilizados. O retalho foi definido como toda sobra com comprimento superior ou igual à média dos itens demandados.

Combinando os parâmetros $K, m, v_{1}$ e $v_{2}$, foram geradas as classes de exemplos apresentadas na Tabela 1.

Os procedimentos computacionais desenvolvidos foram implementados em Dephi 6.0 e executados em um computador Pentium IV (3 GHz com 2 GB de memória RAM). 
Tabela 1 - Descrição das classes.

\begin{tabular}{|c|c|c|c|c||c|c|c|c|c|}
\hline Classe & $K$ & $m$ & $v_{1}$ & $v_{2}$ & Classe & $K$ & $m$ & $v_{1}$ & $v_{2}$ \\
\hline 1 & 5 & 10 & 0,001 & 0,25 & 10 & 7 & 20 & 0,001 & 0,5 \\
\hline 2 & 5 & 10 & 0,001 & 0,5 & 11 & 7 & 40 & 0,001 & 0,25 \\
\hline 3 & 5 & 20 & 0,001 & 0,25 & 12 & 7 & 40 & 0,001 & 0,5 \\
\hline 4 & 5 & 20 & 0,001 & 0,5 & 13 & 9 & 10 & 0,001 & 0,25 \\
\hline 5 & 5 & 40 & 0,001 & 0,25 & 14 & 9 & 10 & 0,001 & 0,5 \\
\hline 6 & 5 & 40 & 0,001 & 0,5 & 15 & 9 & 20 & 0,001 & 0,25 \\
\hline 7 & 7 & 10 & 0,001 & 0,25 & 16 & 9 & 20 & 0,001 & 0,5 \\
\hline 8 & 7 & 10 & 0,001 & 0,5 & 17 & 9 & 40 & 0,001 & 0,25 \\
\hline 9 & 7 & 20 & 0,001 & 0,25 & 18 & 9 & 40 & 0,001 & 0,5 \\
\hline
\end{tabular}

\subsection{Resultados}

Nesta seção, apresentamos os resultados computacionais obtidos pelos procedimentos heurís-

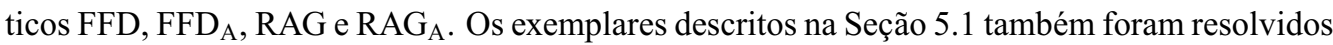
pelo algoritmo COLA, desenvolvido por Gradisar et al. (1997). A Tabela 2 mostra a média da perda relativa nos objetos padronizados (Média Prop), não padronizados (Média Pronp) e retalho líquido relativo (Média Rlr) para os exemplares de todas as classes. Os maiores e menores valores estão em itálico e em negrito, respectivamente. Os resultados médios de cada classe encontramse nas Tabelas A1, A2 e A3 do Apêndice.

Tabela 2 - Perda media relativa nos objetos padronizados, não padronizados e retalho líquido relativo.

\begin{tabular}{|c|c|c|c|c|c|}
\hline & & \multicolumn{2}{|c|}{ Construtiva } & \multicolumn{2}{c|}{ Residual } \\
\cline { 2 - 6 } & COLA & FFD & FFD $_{\text {A }}$ & RAG 1 & RAG $_{\text {A }} 1$ \\
\hline Média Prop & 0,0183 & 0,0088 & $\mathbf{0 , 0 0 0 2}$ & 0,0021 & 0,0005 \\
Média Pronp & 0,0556 & 0,0328 & 0,0090 & 0,0977 & $\mathbf{0 , 0 0 7 0}$ \\
Média Rlr & $\mathbf{0 , 2 5 4 0}$ & 0,6447 & 0,9540 & 0,9110 & 0,9508 \\
\hline
\end{tabular}

Diferentemente dos procedimentos heurísticos utilizados, o algoritmo COLA ordena os objetos de modo não decrescente e, para cada objeto nesta sequência, constrói um padrão de corte que prioriza o uso de objetos não padronizados. Assim, o algoritmo consegue reduzir $75 \%$ do estoque de objetos não padronizados, aproximadamente. As heurísticas propostas para o problema de aproveitamento reduzem gradativamente o estoque de objetos não padronizados, uma vez que o uso desses objetos é estimulado apenas por permitir que uma perda maior em relação aos objetos padronizados seja gerada.

Em relação às perdas, observa-se que as heurísticas orientadas para o aproveitamento de sobras apresentam soluções superiores ao algoritmo COLA e às heurísticas originais (clássicas). Um fato que se deve destacar é que mesmo permitindo uma perda maior nos objetos não padroniza- 
dos (uma maneira destes terem preferência) as soluções apresentadas pelas heurísticas específicas para o problema com sobras aproveitáveis são as que apresentam menores perdas. A redução das perdas deve-se ao fato de que sobras indesejáveis são alteradas para se tornarem uma perda pequena ou um retalho (objeto não padronizado futuro). Entretanto, essa redução pode originar um efeito indesejável para a solução, pois muitos retalhos podem ser gerados. O aumento de objetos não padronizados futuros pode comprometer a qualidade da solução, conforme a Definição 1 , tornando uma solução indesejável ou até mesmo inaceitável.

\section{ANÁLISE DOS MÉTODOS DE SOLUÇÃO}

A classificação dos métodos de solução é realizada aplicando-se o processo de inferência fuzzy (Seção 4), visto que vários fatores são considerados simultaneamente na avaliação das soluções.

Para o processo de inferência fuzzy, utilizou-se o Toolbox Fuzzy Logic do software MatLab 7.1 (Mathworks Inc., 1999). Para a análise dos resultados, foram relacionados os dados da Tabela 2. Estes dados foram escolhidos para a análise da solução final por serem fatores que influenciam diretamente na qualidade da solução, ou seja, o comprimento total perdido está diretamente relacionado com a quantidade de novos retalhos que retornam ao estoque.

Para os parâmetros definidos na Seção 2, consideramos os valores 0,01 e 0,015 para os parâmetros $\xi_{2}^{p}$ e $\xi_{1}^{p}$, respectivamente, e para os parâmetros $\xi_{1}^{n p}$ e $\xi_{2}^{n p}$, definimos os valores 0,015 e 0,03 , respectivamente. Considerando o retalho líquido relativo, definimos $\omega_{1}=0,5, \omega_{2}=0,8 \mathrm{e}$ $\omega_{3}=1,0$.

As variáveis de entrada para o sistema fuzzy proposto são: perda relativa nos objetos padronizados e não padronizados e o retalho líquido relativo. A variável de saída é a classificação das soluções em ideal (ID), desejável (DE), aceitável (AC), indesejável (IND) e inaceitável (INA), conforme a Definição 1. As funções de pertinência relacionadas a esses dados têm como formato os padrões geométricos mostrados nas Figuras 5 e 6 com os parâmetros: MPE (Muito Pequenas), PE (Pequenas) e G (Grandes) para as perdas relativas e BS (Bem Significativas), PS (Pouco Significativas), E (Estável) e A (Ampliado) para os retalho líquido relativo. Na Figura 5, para todos os valores referentes à perda relativa nos objetos padronizados e não padronizados e retalho líquido relativo, adicionou-se a média de seus respectivos desvios-padrões, sendo que:

- Desvio-padrão médio da perda nos objetos padronizados: 0,008;

- Desvio-padrão médio da perda nos objetos não padronizados: 0,061;

- Desvio-padrão médio do retalho líquido relativo: 0,166.

Desta forma, considerando-se o primeiro valor na Figura 5(b) tem-se que 0,666 foi obtido assumindo $\omega_{1}=0,5$ adicionado do desvio padrão 0,166 .

A construção dos padrões geométricos da Figura 5 foi baseada nos valores da Tabela 2. Entretanto, para a construção dos padrões geométricos da Figura 5 (a e c) multiplicou-se os valores obtidos por 100, devido à ordem de grandeza que os mesmos apresentavam. 


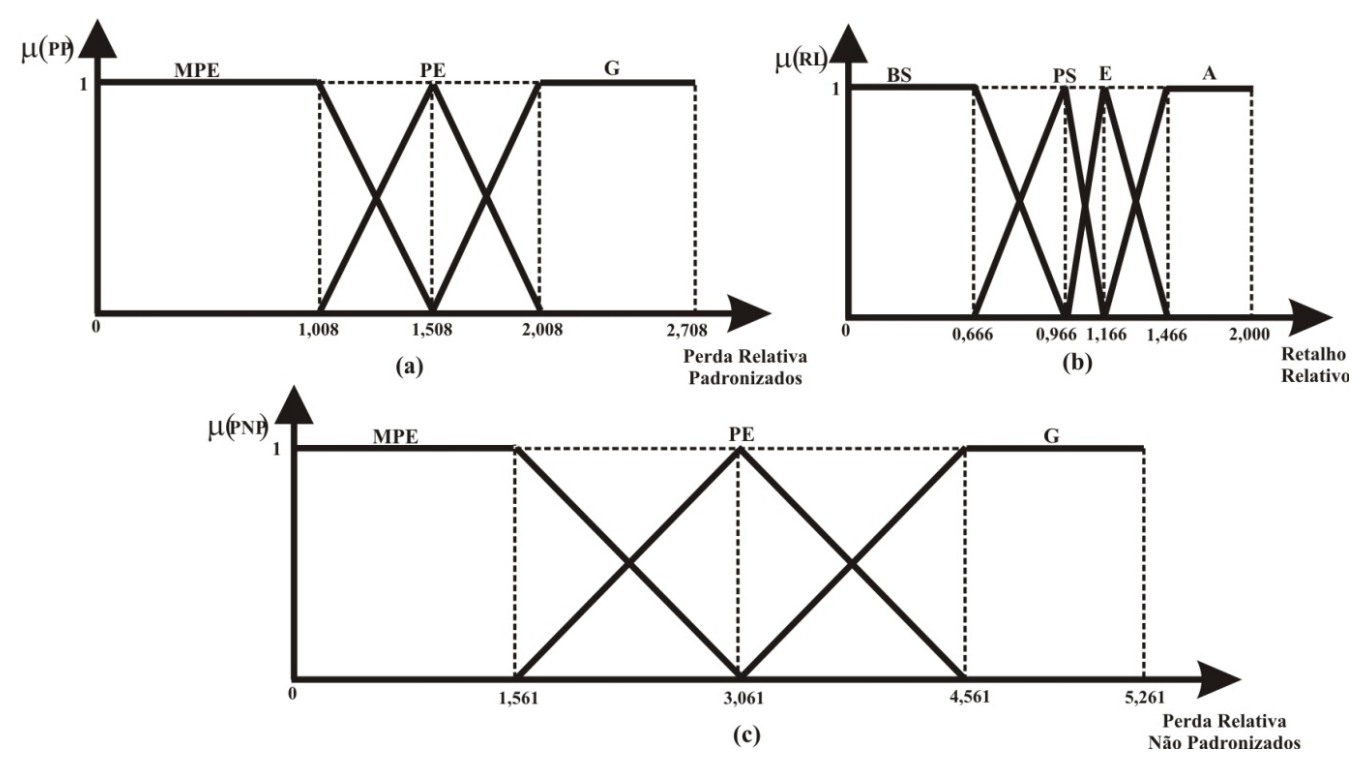

Figura 5 - Padrões geométricos: (a) Perda Relativa nos Objetos Padronizados, (b) Retalho Líquido Relativo, (c) Perda Relativa nos Objetos não Padronizados.

Os padrões geométricos apresentados na Figura 6 são referentes aos dados de saída estabelecidos para o sistema fuzzy.

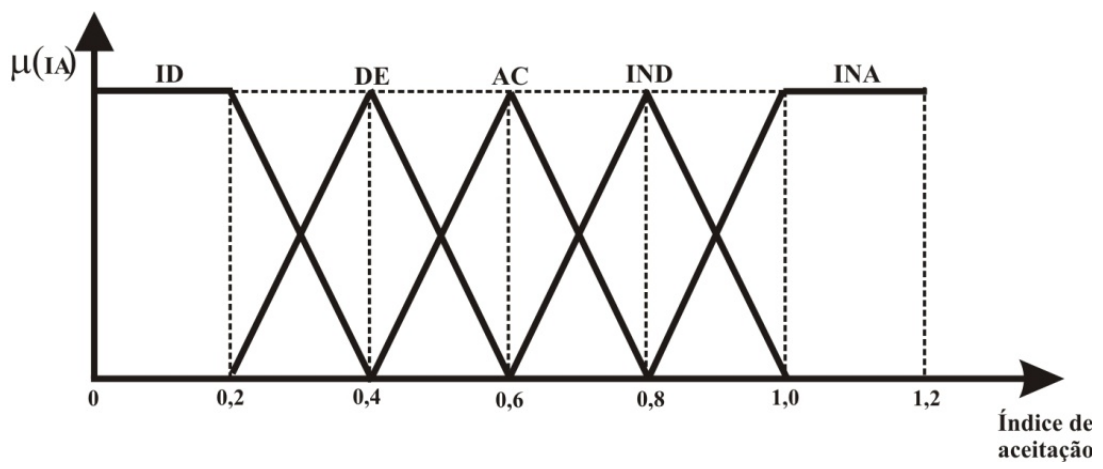

Figura 6 - Saída fuzzy: Índice de aceitabilidade.

Observe que os padrões geométricos apresentados na Figura 6 são igualmente espaçados. Isto ocorre porque no problema de reconhecimento de padrões todas as classes devem ter a mesma probabilidade de serem escolhidas. Desta forma, é usual normalizar a saída de dados (entre 0 e 1 ou outro intervalo conveniente). Ressalta-se que o processo de reconhecimento de padrões é qualitativo, ou seja, o que importa é a classe a qual pertence uma dada solução.

O ajuste entre os padrões geométricos e a saída fuzzy é geralmente feito com base na fixação dos consequentes e na alteração dos antecedentes (funções de pertinência). 
As regras do sistema fuzzy que representam o conhecimento base para análise das soluções do problema têm o seguinte formato:

Regra 1: Se (a perda relativa nos objetos padronizados for muito pequena) e (a perda relativa nos objetos não padronizados for muito pequena) e (a redução de retalhos for bem significativa), então a solução é ideal.

De maneira simplificada, a Tabela 3 sintetiza as 36 possíveis regras fuzzy. Nesta tabela, consideramos PerdaRelPad e PerdaRelNPad a perda relativa nos objetos padronizados e não padronizados, respectivamente. Bem Signif. e Pouco Signif. são referentes aos termos bem significativa e pouco significativa, respectivamente.

Tabela 3 - Regras fuzzy.

\begin{tabular}{|c|c|c|c|c|}
\hline \multirow{2}{*}{$\begin{array}{r}\text { PerdaRelNPad } \\
\text { PerdaRelPad } \\
\end{array}$} & \multicolumn{4}{|c|}{ Redução do Retalho Líquido Relativo } \\
\hline & Bem Signif. & Pouco Signif. & Estável & Ampliado \\
\hline $\begin{array}{l}\text { Muito Pequena } \\
\text { Muito Pequena }\end{array}$ & Ideal & Desejável & Aceitável & Aceitável \\
\hline $\begin{array}{ll}\text { Muito Pequena } & \text { Pequena } \\
\end{array}$ & Desejável & Desejável & Aceitável & Indesejável \\
\hline Muito Pequena & Aceitável & Aceitável & Aceitável & Indesejável \\
\hline Pequena $\quad$ Muito Pequena & Desejável & Aceitável & Aceitável & Indesejável \\
\hline $\begin{array}{ll}\text { Pequena } & \text { Pequena } \\
\end{array}$ & Desejável & Aceitável & Aceitável & Indesejável \\
\hline $\begin{array}{ll}\text { Pequena } & \text { Grande } \\
\end{array}$ & Aceitável & Aceitável & Indesejável & Indesejável \\
\hline Grande $\quad$ Muito Pequena & Aceitável & Aceitável & Indesejável & Indesejável \\
\hline Grande $\quad$ Pequena & Aceitável & Indesejável & Indesejável & Indesejável \\
\hline Grande $\quad$ Grande & Indesejável & Indesejável & Indesejável & Inaceitável \\
\hline
\end{tabular}

Os operadores utilizados em todo processo de inferência são:

- Implicação: Mamdami.

- Agregação: Máximo.

- Defuzzificação: Centro de Área (CDA).

- $\tau$-norma: Mínimo.

Para uma melhor compreensão do desenvolvimento do sistema fuzzy utilizado para classificar os métodos de solução para o PCESA, as etapas do processo desenvolvido são ilustradas a seguir. Para esta ilustração, foram consideradas as 36 regras apresentadas na Tabela 3 e os dados de entrada da heurística $\mathrm{RAG}_{\mathrm{A}}$ (Tabela 2).

Inicialmente, localizam-se as regras que foram ativadas a partir dos dados de entrada da heurística $\mathrm{RAG}_{\mathrm{A}}$ e dos padrões geométricos definidos (Fig. 5). Para os valores fornecidos pela heurística $\mathrm{RAG}_{\mathrm{A}}$, tem-se apenas duas regras ativadas: 
Regra 1: Se (a perda relativa nos objetos padronizados for muito pequena) e (a perda relativa nos objetos não padronizados for muito pequena) e (a redução do retalho líquido relativo for bem significativa), então a solução é ideal.

Regra 2: Se (a perda relativa nos objetos padronizados for muito pequena) e (a perda relativa nos objetos não padronizados for muito pequena) e (a redução do retalho líquido relativo for pouco significativa), então a solução é desejável.

A representação geométrica das regras que foram ativadas são apresentadas na Figura 7. Observe que na representação geométrica do retalho líquido relativo duas regras foram ativadas (BS: bem significativa e PS: pouco significativa).

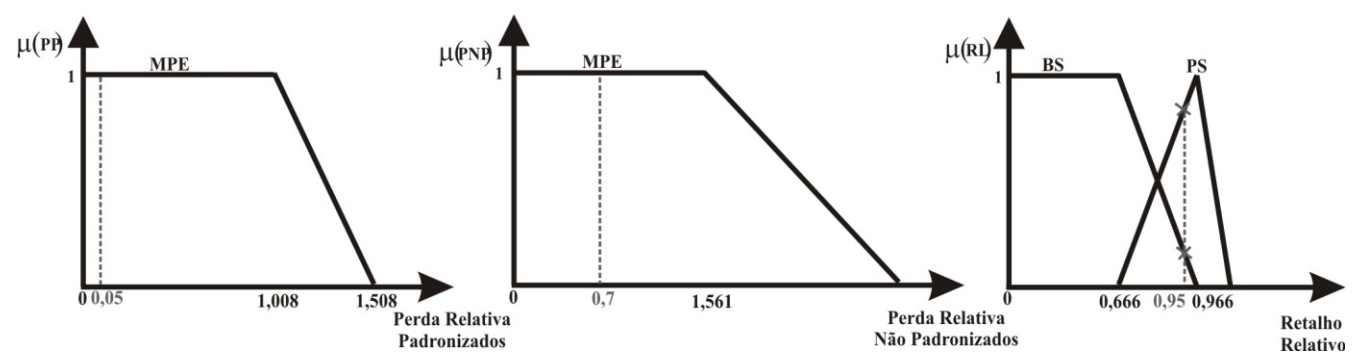

Figura 7 - Ilustração gráfica do processo de Inferência Fuzzy.

O próximo passo consiste em desmembrar os termos das respectivas funções de pertinência envolvidas com as regras ativadas e verificar suas respectivas regiões fuzzy de saída. Estas regiões de saída são obtidas utilizando os padrões geométricos da Figura 6.

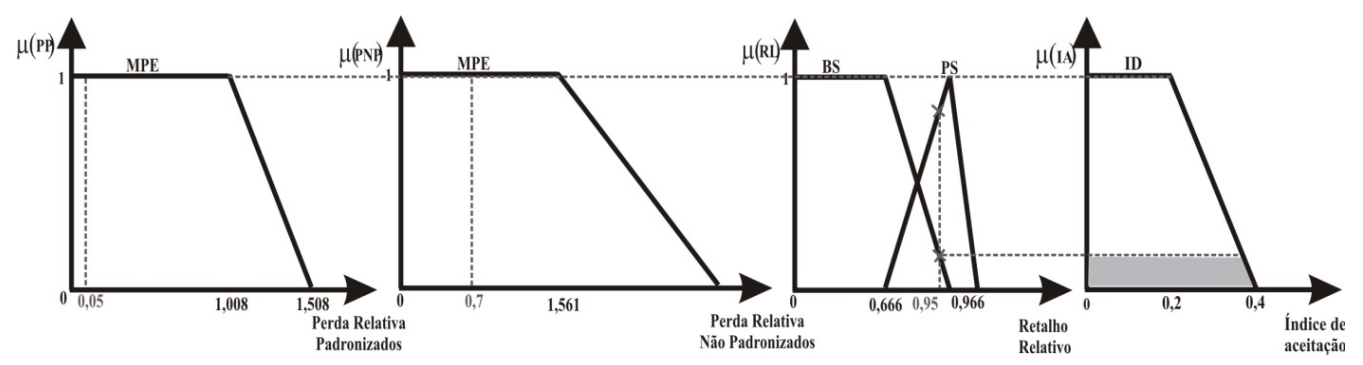

Figura 8 - Região ativada: Regra 1.

Combinando as duas regiões fuzzy de saída, obtém-se uma região fuzzy que representa a agregação das regras ativadas. Para esta agregação, na qual utilizou-se a regra do máximo, tem-se a região apresentada na Figura 10.

Após obter a região resultante da agregação das regras ativadas, aplica-se um método de defuzzificação, que especifica um ponto na saída que melhor representa o conjunto fuzzy. O ponto localizado é um termo comum que combina a contribuição de cada regra ativada. A solução 


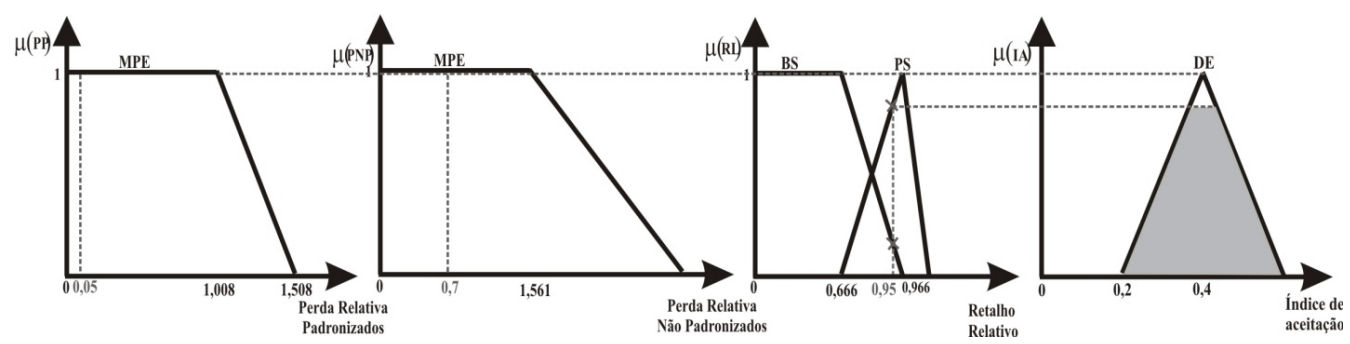

Figura 9 - Região ativada: Regra 2.
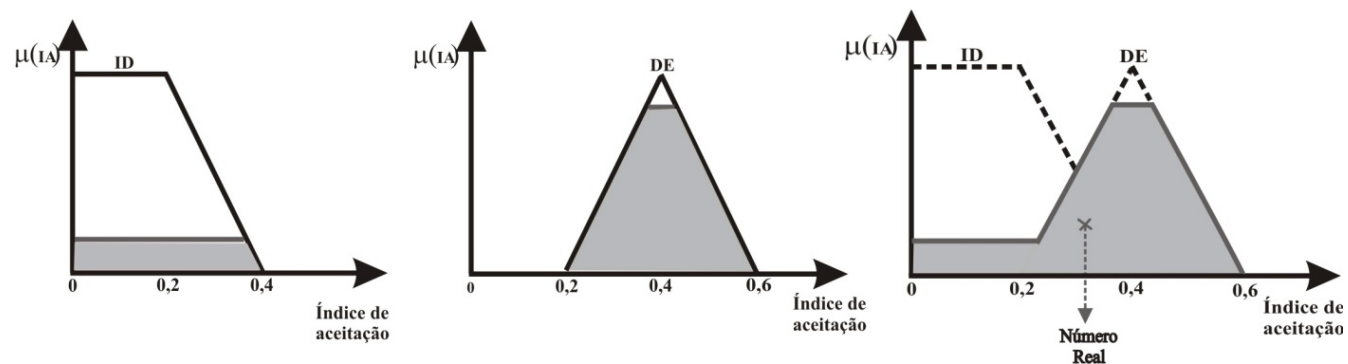

Figura 10 - Ilustração gráfica da região fuzzy de regras ativadas.

obtida pelo processo de defuzzificação é um número real que permite uma análise precisa do resultado.

$\mathrm{Na}$ Tabela 4, apresenta-se o resultado e a classificação das heurísticas analisadas.

Tabela 4 - Classificação das soluções.

\begin{tabular}{|c|c|c|c|c|c|}
\hline & & \multicolumn{2}{|c|}{ Construtiva } & \multicolumn{2}{c|}{ Residual } \\
\cline { 2 - 6 } & COLA & FFD & FFD $_{\text {A }}$ & RAG 1 & RAG $_{\text {A }} 1$ \\
\hline CDA & 0,8 & 0,437 & 0,388 & 0,6 & 0,385 \\
Solução & IND & AC & DE & AC & DE \\
\hline
\end{tabular}

A partir das análises realizadas com o auxílio dos operadores fuzzy, tem-se que as melhores soluções para o problema de corte de estoque com sobras aproveitáveis quando se relaciona a perda relativa nos objetos padronizados e não padronizados e o retalho líquido relativo, foram apresentadas pelas heurísticas Residuais $\mathrm{FFD}_{\mathrm{A}}$ e $\mathrm{RAG}_{\mathrm{A}}$. Assim, podemos dizer que essas heurísticas são as mais indicadas para resolver o PCESA ilustrado nesse trabalho. Observe que, embora não seja ideal, há uma melhora na solução quando comparamos as heurísticas clássicas com sua respectiva versão para o aproveitamento de sobras. Com relação ao algoritmo COLA, temos que ele apresenta uma solução indesejável. Isto ocorre pelo fato deste algoritmo apresentar uma perda relativa grande tanto para os objetos padronizados quanto para os objetos não padronizados. 


\section{CONCLUSÕES}

Neste trabalho, abordou-se o problema de corte de estoque em que as sobras geradas pelo processo produtivo podem ser aproveitadas como objetos não padronizados no atendimento de demandas futuras. Para resolver o PCESA, alguns métodos heurísticos clássicos da literatura foram revisados. Entretanto, a análise dos métodos de solução não é trivial, pois vários parâmetros podem concorrer simultaneamente na determinação da melhor solução e, consequentemente, da melhor estratégia para gerá-la. Por exemplo, os parâmetros perda total, retalho total e número de padrões de corte com perdas pequenas podem, isoladamente, fornecer soluções diferentes segundo a definição proposta, o que ocasiona uma dificuldade adicional no momento de escolher a melhor estratégia numa determinada situação que considere todos esses parâmetros ao mesmo tempo.

Para lidar com esse problema, utilizou-se um mecanismo de inferência fuzzy com a finalidade de realizar uma análise geral dos métodos de solução. Este mecanismo permite que a contribuição de cada característica de interesse na solução seja considerada simultaneamente. A partir do processo de defuzzificação, um valor pontual foi determinado para cada método heurístico proposto, sendo possível classificá-los de acordo com a definição estabelecida a priori. Com os testes computacionais realizados, verificou-se que os procedimentos heurísticos orientados para o problema de aproveitamento de sobras apresentaram soluções superiores aos procedimentos heurístico clássicos, confirmando as conclusões obtidas em Cherri et al. (2009).

Assim, a metodologia fuzzy empregada facilitou a análise dos procedimentos heurísticos desenvolvidos para resolver o PCESA. Apesar da especificidade do PCESA, a metodologia desenvolvida nesse trabalho pode ser aplicada na comparação e classificação de métodos de solução de diversos outros problemas, desde que se tenham vários fatores que concorram na determinação do melhor método ou solução.

Como sugestões de trabalhos futuros, pode-se aplicar a metodologia fuzzy para classificar os métodos heurísticos do PCESA no caso bidimensional. O aproveitamento de sobras em problemas de corte bidimensionais é recente na literatura (Cherri, 2009), entretanto, assim como nos problemas de corte unidimensionais, a determinação da melhor solução envolve a análise simultânea de diversos fatores conflitantes presentes nas soluções.

\section{AGRADECIMENTOS}

Os autores agradecem ao Conselho Nacional de Desenvolvimento Científico e Tecnológico $\mathrm{CNPq}$ - pelo apoio financeiro. Os autores também gostariam de agradecer ao professor Marcos Arenales, que contribuiu para o desenvolvimento desse trabalho.

\section{REFERÊNCIAS}

[1] Abuabara A \& Morabito R. 2009. Cutting optimization of structural tubes to build agricultural light aircrafts. Annals of Operations Research, 169: 149-165. 
[2] Arenales MN, Morabito R \& Yanasse H. (eds.) 1999. Cutting and packing problems. Pesquisa Operacional, 19(2).

[3] Bischoff E \& Wäscher G. (EDS.) 1995. Cutting and packing. European Journal of Operational Research, 84(3): special issue.

[4] BROWN AR. 1971. Optimum packing and depletion: the computer in space and resource usage problem. New York: Macdonald - London and American Elsevier Inc.

[5] Cherri AC, Arenales MN \& Yanasse HH. 2009. The one dimensional cutting stock problems with usable leftover: A heuristic approach. European Journal of Operational Research, 196: 897908.

[6] DOLAN E \& MORÉ J. 2002. Benchmarking optimization software with performance profiles. Mathematical. Programming Ser. A, 91: 201-213.

[7] Dowsland K \& Dowsland W. 1992. Packing problems. European Journal of Operational Research, 56: $2-14$.

[8] Dyckhoff H. 1981. A new linear programming approach to the cutting stock problem. Operations Research, 29: 1092-1104.

[9] Dyckhoff H, Kruse HJ, Abel D \& Gal T. 1985. Trim loss and related problems. The International Journal of Management Science, 13: 59-72.

[10] Dyckhoff H \& WÄscher G. (EDS.) 1990. Cutting and packing. European Journal of Operational Research, 44(2): special issue.

[11] ESiCUP - Euro Special Interest Group on Cutting And Packing. Available in: http://www.fe.up.pt/esicup/ (accessed in 2009).

[12] Ghodsi R \& SAsSani F. 2002. An adaptive fuzzy algorithm for cut sequencing of solid wood in furniture component production. Intelligent Control. Proceedings of the 2002 IEEE International Symposium, 246-251.

[13] GHOdsi R \& SASSANi F. 2005. Real-time optimum sequencing of wood cutting process. International Journal of Production Research, 4(6): 1127-1141.

[14] GILMORE PC \& GOMORY RE. 1961. A linear programming approach to the cutting stock problem. Operations Research, 9: 848-859.

[15] Gilmore PC \& GOMORY RE. 1963. A linear programming approach to the cutting stock problem - Part II. Operations Research, 11: 863-888.

[16] Gradisar M, Jesenko J \& Resinovic C. 1997. Optimization of roll cutting in clothing industry. Computers \& Operations Research, 10: 945-953.

[17] Gradisar M, Kljajic M, Resinovic C \& Jesenko J. 1999. A sequential heuristic procedure for one-dimensional cutting. European Journal of Operational Research, 114: 557-568.

[18] GUiffrida AL \& NAGi R. 1998. Fuzzy set theory applications in production management research: a literature survey. Journal of Intelligent Manufacturing, 9: 39-56.

[19] HIFI M. (ED.) 2002. Cutting and packing problems. Studia Informatica Universalis, 2(1): 1-161, special issue.

[20] HinXman A. 1980. The trim-loss and assortment problems: a survey. European Journal of Operational Research, 5: 8-18. 
[21] Mathworks Inc. 1999. Fuzzy Logic Toolbox User's Manual, Natick, MA, USA. Disponível em http://som.yale.edu/unix/research_computing/pdf_doc/fuzzy/fuzzy_tb.pdf.

[22] Oliveira JF \& WÄScher G. (EDS.) 2007. Special Issue on Cutting and Packing. European Journal of Operational Research, 183.

[23] Pedrycz W \& Gomide FC. 1998. An Introduction to Fuzzy Sets: Analysis and Design. The MIT Press, Massachusetts.

[24] Poldi KC \& Arenales MN. 2009. Heuristics for the one-dimensional cutting stock problem with limited multiple stock lengths. Computers and Operations Research, 36: 2074-2081.

[25] Roodman GM. 1986. Near-optimal solutions to one-dimensional cutting stock problem. Computers and Operations Research, 13: 713-719.

[26] SAAd OM, El-Shafei MK \& EzZAT LE. 2007. On treating multiobjective cutting stock problem in the aluminum industry under fuzzy environment. Trends in Applied Sciences Research, 2: 374-385.

[27] Scheithauer G. 1991. A note on handling residual length. Optimization, 22: 461-466.

[28] Sinuany-Stern Z \& Weiner I. 1994. The one dimensional cutting stock problem using two objectives. Journal of Operations Research Society, 45: 231-236.

[29] Sweeney P \& PARTERnoster E. 1992. Cutting and packing problems: a categorized, applicationoriented research bibliography. Journal of Operational Research Society, 43: 691-706.

[30] VAsko FL, Wolf FE \& StotT KL. 1989. A practical solution to a fuzzy two-dimensional cutting stock problem. Fuzzy Sets and Systems, 29: 259-275.

[31] ZADEH LA. 1965. Fuzzy Sets. Information and Control, 8: 338-353.

[32] WANG DX. 1996. A course in Fuzzy Systems and control, Prentice-Hall, Inc., Upper Saddle River, New Jersey.

[33] WANG PY \& Wëscher G. 2002. Cutting and packing. European Journal of Operational Research, 141: $239-469$

[34] WÄSCher G, Haussner H \& SCHUMANn H. 2007. An improved typology of cutting and packing problems. European Journal of Operational Research, 183: 1109-1130. 


\section{APÊNDICE}

Tabela A1 - Perda média relativa nos objetos padronizados.

\begin{tabular}{|l||l||l|l||l|l|}
\hline \multicolumn{1}{|c||}{} & \multicolumn{1}{c||}{} & \multicolumn{2}{c||}{ Construtiva } & \multicolumn{2}{c|}{ Residual } \\
\cline { 2 - 6 } & COLA & FFD & FFD $_{\mathrm{A}}$ & RAG 1 & RAG $_{\mathrm{A}} 1$ \\
\hline Classe 1 & 0,0016 & 0,0039 & 0,0003 & 0,0020 & 0,0007 \\
Classe 2 & 0,0257 & 0,0237 & 0,0005 & 0,0085 & 0,0020 \\
Classe 3 & 0,0115 & 0,0031 & 0,0001 & 0,0014 & 0,0001 \\
Classe 4 & 0,0492 & 0,0103 & 0,0004 & 0,0029 & 0,0003 \\
Classe 5 & 0,0037 & 0,0012 & 0,0000 & 0,0001 & 0,0000 \\
Classe 6 & 0,0340 & 0,0066 & 0,0002 & 0,0007 & 0,0000 \\
Classe 7 & 0,0000 & 0,0033 & 0,0001 & 0,0009 & 0,0003 \\
Classe 8 & 0,0194 & 0,0269 & 0,0006 & 0,0082 & 0,0021 \\
Classe 9 & 0,0021 & 0,0021 & 0,0001 & 0,0001 & 0,0000 \\
Classe 10 & 0,0388 & 0,0119 & 0,0004 & 0,0020 & 0,0004 \\
Classe 11 & 0,0114 & 0,0018 & 0,0000 & 0,0000 & 0,0000 \\
Classe 12 & 0,0382 & 0,0091 & 0,0002 & 0,0009 & 0,0000 \\
Classe 13 & 0,0000 & 0,0056 & 0,0001 & 0,0002 & 0,0002 \\
Classe 14 & 0,0066 & 0,0217 & 0,0004 & 0,0077 & 0,0024 \\
Classe 15 & 0,0005 & 0,0021 & 0,0000 & 0,0001 & 0,0001 \\
Classe 16 & 0,0214 & 0,0163 & 0,0002 & 0,0013 & 0,0002 \\
Classe 17 & 0,0131 & 0,0014 & 0,0000 & 0,0000 & 0,0000 \\
Classe 18 & 0,0531 & 0,0067 & 0,0002 & 0,0004 & 0,0000 \\
\hline Média & 0,0183 & 0,0088 & 0,0002 & 0,0021 & 0,0005 \\
\hline
\end{tabular}

Tabela A2 - Perda média relativa nos objetos não padronizados.

\begin{tabular}{|l||l||c|c||c|c|}
\hline \multicolumn{1}{|c||}{} & \multicolumn{1}{|c||}{} & \multicolumn{2}{c||}{ Construtiva } & \multicolumn{2}{c|}{ Residual } \\
\cline { 2 - 6 } & COLA & FFD & FFD $_{\text {A }}$ & RAG 1 & RAG $_{\text {A }} 1$ \\
\hline Classe 1 & 0,0810 & 0,0517 & 0,0053 & 0,0933 & 0,0048 \\
Classe 2 & 0,0954 & 0,0736 & 0,0102 & 0,1000 & 0,0108 \\
Classe 3 & 0,0252 & 0,0349 & 0,0047 & 0,0872 & 0,0082 \\
Classe 4 & 0,0619 & 0,0450 & 0,0165 & 0,0909 & 0,0093 \\
Classe 5 & 0,0008 & 0,0082 & 0,0034 & 0,0914 & 0,0052 \\
Classe 6 & 0,0074 & 0,0190 & 0,0157 & 0,1409 & 0,0090 \\
Classe 7 & 0,0953 & 0,0531 & 0,0086 & 0,1045 & 0,0064 \\
Classe 8 & 0,0781 & 0,0648 & 0,0122 & 0,1108 & 0,0100 \\
Classe 9 & 0,0555 & 0,0301 & 0,0052 & 0,0921 & 0,0040 \\
Classe 10 & 0,0830 & 0,0369 & 0,0136 & 0,0933 & 0,0077 \\
Classe 11 & 0,0056 & 0,0100 & 0,0025 & 0,0778 & 0,0061 \\
Classe 12 & 0,0218 & 0,0164 & 0,0136 & 0,1418 & 0,0054 \\
Classe 13 & 0,1122 & 0,0338 & 0,0058 & 0,0975 & 0,0060 \\
Classe 14 & 0,0559 & 0,0447 & 0,0136 & 0,0586 & 0,0086 \\
Classe 15 & 0,0805 & 0,0157 & 0,0052 & 0,0725 & 0,0039 \\
Classe 16 & 0,0832 & 0,0275 & 0,0129 & 0,1227 & 0,0077 \\
Classe 17 & 0,0162 & 0,0081 & 0,0024 & 0,0734 & 0,0050 \\
Classe 18 & 0,0417 & 0,0166 & 0,0107 & 0,1108 & 0,0072 \\
\hline Média & 0,0556 & 0,0328 & 0,0090 & 0,0977 & 0,0070 \\
\hline
\end{tabular}

Tabela A3 - Retalho Líquido Relativo.

\begin{tabular}{|l||l||l|l||l|l|}
\hline \multicolumn{1}{|c||}{} & \multicolumn{1}{|c||}{} & \multicolumn{2}{c||}{ Construtiva } & \multicolumn{2}{c|}{ Heurística } \\
\cline { 2 - 6 } & COLA & FFD & FFD $_{\mathrm{A}}$ & RAG 1 & RAG $_{\mathrm{A}} 1$ \\
\hline Classe 1 & 0,3448 & 0,6818 & 0,8949 & 0,8627 & 0,9061 \\
Classe 2 & 0,3347 & 0,6060 & 1,1075 & 0,9023 & 1,0032 \\
Classe 3 & 0,0836 & 0,6086 & 0,8555 & 0,8872 & 0,9029 \\
Classe 4 & 0,1226 & 0,5089 & 1,0907 & 0,9193 & 0,9971 \\
Classe 5 & 0,0637 & 0,5119 & 0,8219 & 0,8691 & 0,9011 \\
Classe 6 & 0,0703 & 0,4291 & 1,1449 & 0,8851 & 0,9723 \\
Classe 7 & 0,4769 & 0,7662 & 0,9009 & 0,9130 & 0,9333 \\
Classe 8 & 0,5583 & 0,7373 & 1,0311 & 0,9227 & 0,9801 \\
Classe 9 & 0,2199 & 0,7220 & 0,9037 & 0,9112 & 0,9393 \\
Classe 10 & 0,1949 & 0,5775 & 1,0005 & 0,9160 & 0,9739 \\
Classe 11 & 0,0375 & 0,6214 & 0,8377 & 0,9115 & 0,9405 \\
Classe 12 & 0,0687 & 0,5368 & 1,0005 & 0,9190 & 0,9696 \\
Classe 13 & 0,5913 & 0,8246 & 0,9171 & 0,9243 & 0,9439 \\
Classe 14 & 0,6058 & 0,7840 & 0,9650 & 0,9190 & 0,9451 \\
Classe 15 & 0,3590 & 0,7669 & 0,9106 & 0,9313 & 0,9446 \\
Classe 16 & 0,3068 & 0,6367 & 0,9541 & 0,9234 & 0,9493 \\
Classe 17 & 0,0631 & 0,6830 & 0,8838 & 0,9342 & 0,9481 \\
Classe 18 & 0,0693 & 0,6019 & 0,9511 & 0,9468 & 0,9650 \\
\hline Média & 0,2540 & 0,6447 & 0,9540 & 0,9110 & 0,9508 \\
\hline
\end{tabular}

